\title{
CDK inhibitors in hormone receptor positive advanced breast cancer
}

Bilge AKTAS

\begin{abstract}
Endocrine treatment is the current first line treatment in ER+ advanced breast cancer with a requirement of predictive biomarkers to the treatment and different approaches for endocrine resistance risk. Cyclin dependent kinases (CDKs) are regulating each step of cellular division in mammalian cell cycle and are hyperactivated in cancer cells causing cancer progression. Next generation pan-CDK inhibitors are in clinical development, while clinical trials in breast cancer, particularly in ER+ subtype where the greatest growth inhibition has been observed. Here I will review the data from this new class of drugs.
\end{abstract}

Keywords: CDK inhibitors, Hormone positive advanced breast cancer, Targeted therapies, Palbociclib, Endocrine therapy resistance

\section{Introduction}

Hormone receptor positive (HR+) breast cancer is the most commonly diagnosed molecular subset of advanced breast cancer and benefits highly from endocrine treatment in both adjuvant and metastatic setting with a relatively low toxicity profile [1]. Despite having an indolent course it remains incurable. The current goals of therapy in metastatic breast cancer are maintaining a good quality of life, prolonging the survival and delaying initiation of chemotherapy [2].

Bilge Aktas, M.D., ( $($ )

Section of Medical Oncology, Yale Cancer Center, Yale School of Medicine, 333 Cedar Street, New Haven, CT 06520, USA

e-mail:bilge.aktas@yale.edu
Several types of single-agent or combined endocrine treatment modalities are available achieving most of these goals and represent the standard of care for the first line therapy [3]. Nevertheless de novo or acquired resistance develops ultimately with each endocrine regimen. At that point, the patients are usually prone to receive chemotherapy that has little effectiveness and a significant toxicity in this subset of patients.

Unfortunately, lack of predictive biomarkers identifying subgroups of patients that will benefit from endocrine therapy at most is still the current problem addressing future options with combined therapies including the new biological agents. Targeting critical pathways to improve the efficacy of endocrine treatment are in development. The cyclin dependent kinases (CDKs) are a large family of serine threonine kinases interacting with cyclins have a key role in maintaining the control of progression through cell cycle. Since the dysregulation of the cell cycle is a hallmark of unrestricted growth in cancer with a high metastatic potential [4], novel agents targeting CDK pathway has been an attractive option for new treatment modalities [5] .

\section{The biology of CDKs in cancer}

The CDKs are regulating the each step of cellular division in mammalian cell cycle and are hyperactivated in cancer cells causing cancer progression. There are 2 groups of CDKs based on their activity in cell-cycle progression or transcription. Most of the human cells have diploid DNA content exiting the cell cycle and are maintained in a quiescent $\mathrm{G} 0$ state. Extracellular signals including activation by peptide growth factors (such as RAS, MAPK and mTOR) and nuclear receptors (such as estrogen receptor), drive the cell cycle into G1 phase and then S phase from quiescent G0 
phase [6-9]. This occurs mainly by association with D-type cyclins (Cyclin D1, cyclin D2 and cyclin D3) through the regulation of CDK4 or CDK6 complex that are structurally related, similar proteins [10-12]. Cyclin D1 is the best characterized D-type cyclin that is expressed to promote the activity of CDK4 and CDK6 [13].

In contrast to other CDKs, cyclin association is controlled by multiple mechanisms in CDK4 and CDK6. Overexpression/induction of INK4 proteins including p16, p15, p18 and p19 function as inhibitors of CDK4 and CDK6 in response to stress conditions causing G1 arrest of the cell cycle $[7,14,15]$. Similar to other CDKs, CDK4 and CDK6 are subjected to phosphoregulation with a unique preference to the phosphorylation of the tumor suppressor retinoblastoma protein $(\mathrm{RB})$ and the related proteins (RBL1 and RBL2) [16, 17]. RB functions as a multiprotein complex in cell cycle by binding to E2F transcription factors and suppressing transcription of the genes that are regulated by E2Fs. Thus, the phosphorylation of RB by CDK4 and CDK6 causes the release of E2F initiating the progression of cell cycle $[18,19]$.

Deregulation of cyclin D1, RB, INK4 proteins as well as CDK4 and CDK6 can occur in various types of cancers. Importantly, distinct mechanisms of CDK4/6RB-p16 pathway dysregulation is mutually exclusive and are frequently tumor-type specific [20]. Cancer Genome Atlas demonstrated the aberrations causing hyperactivation of cyclin D1-CDK4/6 in particularly ER+ breast cancers [21]. Furthermore, cyclin D1 has been shown to activate ER in the absence of estrogen playing a role in endocrine resistance [22]. In addition, alterations in p16, increase in RB phosphorylation may be associated with resistance to endocrine treatment in breast cancer patients providing a strong rationale for targeting of the pathway [23-26].

\section{Targeted inhibition of CDK4 and CDK6}

CDK inhibitors have two main classes based on their target range: 1 . Broad range $\mathrm{CDK}$ inhibitors act on a variety of CDKs including CDK4/CDK6; 2. Cell-cycle specific inhibitors act on CDK4/CDK6. CDKs mainly block the ATP binding pocket. Early efforts to target CDKs initially started with pan-CDK inhibitors causing a notable toxicity although activity has been observed in hematological/ solid malignancies with a lack of appropriate patient selection [27]. Next generation pan-CDK inhibitors are in clinical development, while clinical trials in breast cancer, particularly in ER+ subtype where the greatest growth inhibition has been observed, are focused on inhibiting cyclinD1-CDK4/6 interaction. Elevated RB and cyclin
D1 or decreased p16 seem to be promising predictors of response in these clinical trials while lack of RB function (loss/inactivation) can cause resistance to CDK4/6 inhibitors since the inhibition depends on downstream of RB in several cancer types [20, 28, 29].

\section{Endocrine therapy in combination with CDK inhibitors}

Resistance to existing endocrine therapies remains a major challenge with recent advances in clarifying molecular mechanisms in disease progression including cross-talk between estrogen receptor (ER) and various growth factor receptor and/or intracellular signaling pathway. Therefore, to improve the efficacy of endocrine treatment by combining targeted agents with endocrine therapy is currently being evaluated with numerous agents including mTOR, PI3K, Akt, cyclin-dependent kinase 4/6, SRC and histone deacetylase inhibitors [30,31]. Preclinical data suggests that targeting mTOR or cyclin-dependent kinase 4/6 (CDK4/6) pathway might increase the endocrine sensitivity $[32,33]$ carrying over these drugs to clinical trials.

Addition of everolimus, an mTOR inhibitor, to endocrine therapy (exemestane) significantly improved progressionfree survival (PFS) of $\mathrm{HR}+$ advanced breast cancers in BOLERO-2 trial (6.9 vs. 2.8 months, hazard ratio $0.43,95 \%$ CI 0.35-0.54; $\mathrm{p}<0.001$ ) [34] while combination of another mTOR inhibitor, temsirolimus, and endocrine therapy did not improve PFS in HORIZON study [30, 35, 36]. Although it seems to be unclear whether addition of mTOR inhibitors should be combined with endocrine therapy to restore endocrine treatment sensitivity, everolimus is approved for aromatase inhibitor (AI) resistant advanced disease based on BOLERO-2 trial [34, 35, 37, 38]. Several PI3K inhibitors are in clinical development with a recent non-significant improvement in PFS in HR+ AI resistant metastatic breast cancer [39, 40]. Three oral agents selectively targeting CDK4/6 developed recently are palbociclib, abemaciclib and LEE011 [1, 36] and are currently under clinical development:

\section{Palbociclib}

The Phase II, randomized PALOMA-1/TRIO-18 trial was designed to evaluate safety and efficacy of palbociclib in combination with letrozole and letrozole alone in $\mathrm{HR}+$ HER 2 negative advanced breast cancer. Patients entered in two cohorts, cohort $1(\mathrm{~N}=66)$ was unselected for potential biomarkers, cohort $2(\mathrm{~N}=99)$ selected for tumor cyclin $\mathrm{D}$ amplification and/or loss of p16. The study has shown a 
significantly improved PFS in the combination arm (20.2 vs. 10.2 months; HR, 0.488; 95\% CI, 0.319-0.748; onesided $\mathrm{P}=0.0004$ ) giving rise to the approval of the drug by FDA in US recently $[35,41]$. Evaluation of cohorts 1 and 2 found improvements in both groups with the addition of palbociclib, but no apparent correlation between presence of biomarker and improved outcomes (cohort 1, PFS 5.7 vs 26.1 month, HR 0.299, $\mathrm{p}<0.0001$; cohort 2 PFS 11.1 vs 18.1 month, HR $0.508, p=0.0045$ ). No improvement in overall survival was observed in this small population (OS 33.3 months letrozole vs 37.5 months letrozole and palbociclib, HR 0.813, $\mathrm{p}=0.2105$ ). Side effects were mainly hematological, with grade 3/4 neutropenia and leucopenia reported in $54 \%$ and $19 \%$ of patients receiving palbociclib, respectively. The cumulative incidence of neutropenia remained stable (75.8 \%) after 2 years follow-up, suggesting the hematologic toxicity occurs early in treatment and no data support long-term or cumulative hematologic toxicity after the exposure [35]. Phase III development is underway worldwide investigating its use as first-line treatment in advanced breast cancer, as well as treatment of recurrent advanced breast cancer and high-risk (PALOMA-2, PALOMA-3, PEARL studies), early-stage breast cancer (PENELOPE-B, DFHCC 13-559 studies) [1, 41].

\section{Abemaciclib}

Preclinical data for abemaciclib have shown activity as both monotherapy and in combination with chemotherapy, as well as possible passage across the blood-brain barrier compared to palbociclib $[42,43]$. But, no biomarkers except hormone positivity were predictive of response, including evaluation of cyclin D1 and Rb in Phase I trials. Phase II/III studies offer aromatase inhibitor with or without abemaciclib in the firstline setting, and post progression on endocrine therapy. A phase II study, MONARCH, is evaluating abemaciclib monotherapy in post-progression on chemotherapy in the metastatic setting [1].

\section{LEE011}

Hematologic and GI toxicity with LEE011 appear to be less than the other two agents in phase I trials [44]. Phase III trial MONALEESA-2 randomized patients to letrozole with or without LEE011 in the first-line setting of metastatic HR+/HER2-disease. In the preoperative setting, MONALEESA-1 is evaluating the contribution of LEE011 to neoadjuvant aromatase inhibitor. A randomized phase $\mathrm{Ib} /$ II study of LEE011 with the PI3K inhibitor BYL719 and letrozole (NCT01872260), and a randomized phase Ib/II study of LEE011 with the mTOR inhibitor everolimus and exemestane (NCT01857193) in pretreated metastatic HR+ breast cancer are ongoing.

\section{Conclusions and future directions}

Endocrine treatment is the current first line treatment in ER+ advanced breast cancer with a requirement of predictive biomarkers to the treatment and different approaches for endocrine resistance risk. Accumulating preclinical data have shown that inhibition of CDK4/6 pathway has an impact on decreasing breast cancer progression through the loss of cell cycle control. Since the early clinical data have suggested the benefit of CDK4/6 inhibition as monotherapy and combined therapy with endocrine treatment modalities in $\mathrm{ER}+$ breast cancer, predictive biomarker aside from estrogen receptor for CDK4/6 inhibition needs to be identified including amplification or overexpression of cyclin D and/ or RB. Studies investigating both poly-endocrine treatment and new agents in combination with endocrine treatment are ongoing and needs a careful consideration against additional toxicity and cost. Hematological and gastrointestinal toxicities seems to be common, but manageable supporting their use in the clinic safely. In addition, investigation of primary and secondary resistance mechanism of the drug will be important for the selection of best population who may benefit from these drugs and further treatment modalities or drug development strategies. Depending on the fact that CDK4/6 inhibitors may be an option for patients who are resistant to endocrine treatment, substantial efforts in clinical trials are ongoing in both advanced and early stage breast cancer treatment.

\section{References}

1. Mayer EL, Targeting breast cancer with CDK inhibitors. Curr Oncol Rep 2015;17:443. doi: 10.1007/s11912015-0443-3

2. Gluck S, Extending the clinical benefit of endocrine therapy for women with hormone receptor-positive metastatic breast cancer: differentiating mechanisms of action. Clin Breast Cancer 2014; 14:75-84. doi: 10.1016/j.clbc.2013.10.008

3. Cardoso F, Costa A, Norton L, et al. ESO-ESMO 2nd international consensus guidelines for advanced breast cancer (ABC2)dagger. Ann Oncol 2014, 25:1871-88. doi: 10.1093/annonc/mdu385

4. Hanahan D, Weinberg RA: Hallmarks of cancer: the next generation. Cell 2011; 144(5):646-674. doi: 10.1016/j.cell.2011.02.013.

5. Malumbres M, Barbacid M. Cell cycle, CDKs and cancer: a changing paradigm. Nature Rev Cancer 
2009;9:153-66. doi: 10.1038/nrc2602.

6. Sherr CJ. Cancer cell cycles. Science 1996; 274(5293):1672-7.

7. Lim S, Kaldis P, Cdks, cyclins and CKIs: roles beyond cell cycle regulation. Development 2013, 140:3079-93. doi: 10.1242/dev.091744

8. Malumbres M, Barbacid M. To cycle or not to cycle: a critical decision in cancer. Nature Rev Cancer 2001;1:222-31.

9. Rodgers JT, King KY, Brett JO, et al. mTORC1 controls the adaptive transition of quiescent stem cells from G0 to G(Alert). Nature 2014; 510:393-96. doi: 10.1038/ nature 13255

10. Sherr CJ, D-type cyclins. Trends in Biochemical Sciences 1995; 20:187-90.

11. Diehl JA, Cycling to cancer with cyclin D1. Cancer Biol Ther 2002; 1:226-31.

12. Pavletich NP, Mechanisms of cyclin-dependent kinase regulation: structures of Cdks, their cyclin activators, and Cip and INK4 inhibitors. J Mol Biol 1999; 287:821-8.

13. Matsushime H, Roussel MF, Ashmun RA, Sherr CJ. Colony-stimulating factor 1 regulates novel cyclins during the G1 phase of the cell cycle. Cell 1991, 65:70113.

14. Witkiewicz AK, Knudsen KE, Dicker AP, Knudsen ES. The meaning of p16(ink4a) expression in tumors: functional significance, clinical associations and future developments. Cell Cycle 2011; 10:2497-503. doi: 10.4161/cc.10.15.16776

15. Serrano M, Hannon GJ, Beach D. A new regulatory motif in cell-cycle control causing specific inhibition of cyclin D/CDK4. Nature 1993, 366:704-7.

16. Kato J, Matsushime H, Hiebert SW, Ewen ME, Sherr CJ. Direct binding of cyclin $\mathrm{D}$ to the retinoblastoma gene product $(\mathrm{pRb})$ and $\mathrm{pRb}$ phosphorylation by the cyclin D-dependent kinase CDK4. Genes and Development 1993, 7:331-42.

17. Matsushime H, Quelle DE, Shurtleff SA, Shibuya M, Sherr CJ, Kato JY. D-type cyclin-dependent kinase activity in mammalian cells. Mol Cell Biol 1994, 14:2066-76.

18. Wang JY, Knudsen ES, Welch PJ. The retinoblastoma tumor suppressor protein. Advances Cancer Research 1994;64:25-85.

19. Burkhart DL, Sage J. Cellular mechanisms of tumour suppression by the retinoblastoma gene. Nature Rev
Cancer 2008; 8:671-82. doi: 10.1038/nrc2399

20. Dickson MA, Molecular pathways: CDK4 inhibitors for cancer therapy. Clin Cancer Res 2014; 20:3379-83. doi: 10.1158/1078-0432.CCR-13-1551

21. Finn RS, Dering J, Conklin D, et al. PD 0332991, a selective cyclin D kinase 4/6 inhibitor, preferentially inhibits proliferation of luminal estrogen receptorpositive human breast cancer cell lines in vitro. Breast Cancer Research:BRC 2009;11:R77. doi: 10.1186/ bcr2419.

22. Zwijsen RM, Wientjens E, Klompmaker R, van der Sman J, Bernards R, Michalides RJ. CDK-independent activation of estrogen receptor by cyclin D1. Cell 1997;88:405-15.

23. Buckley MF, Sweeney KJ, Hamilton JA, et al. Expression and amplification of cyclin genes in human breast cancer. Oncogene 1993, 8:2127-33.

24. Hui R, Macmillan RD, Kenny FS, Musgrove EA, Blamey RW, Nicholson RI, Robertson JF, Sutherland RL. INK4a gene expression and methylation in primary breast cancer: overexpression of p16INK4a messenger RNA is a marker of poor prognosis. Clin Cancer Res 2000;6:2777-87.

25. Watts CK, Brady A, Sarcevic B, deFazio A, Musgrove EA, Sutherland RL, Antiestrogen inhibition of cell cycle progression in breast cancer cells in associated with inhibition of cyclin-dependent kinase activity and decreased retinoblastoma protein phosphorylation. Mol Endocrinol 1995; 9:1804-13.

26. Thangavel C, Dean JL, Ertel A, Knudsen KE, Aldaz CM, Witkiewicz AK, Clarke R, Knudsen ES, Therapeutically activating RB: reestablishing cell cycle control in endocrine therapy-resistant breast cancer. EndocrineRelated Cancer 2011; 18:333-45. doi: 10.1530/ERC10-0262.

27. Casimiro MC, Velasco-Velazquez M, Aguirre-Alvarado C, Pestell RG, Overview of cyclins D1 function in cancer and the CDK inhibitor landscape: past and present. Expert Opin Invest Drugs 2014; 23:295-304. doi: $10.1517 / 13543784.2014 .867017$

28. Gopalan PK G-VA, Zajac-Kaye M, Kaye FJ, Inhibitory effect of the CDK4/6 inhibitor, PD 0332991, is enhanced by mTOR inhibition in non-small cell lung cancer (NSCLC). In: AACR: 2013 Apr 6-10; Washington, DC. Philadelphia (PA): Cancer Res 2013 Apr 6-10.

29. Wiest T, Schwarz E, Enders C, Flechtenmacher C, Bosch FX. Involvement of intact HPV16 E6/E7 gene expression in head and neck cancers with unaltered p53 
status and perturbed $\mathrm{pRb}$ cell cycle control. Oncogene 2002,21:1510-17.

30. Jerusalem G, Bachelot T, Barrios C, Neven P, Di Leo A, Janni W, de Boer R: A new era of improving progressionfree survival with dual blockade in postmenopausal HR(+), HER2(-) advanced breast cancer. Cancer Treat Rev 2015;41:94-104. doi: 10.1016/j.ctrv.2014.12.011

31. Yardley DA, Ismail-Khan RR, Melichar B, et al. Randomized phase II, double-blind, placebo-controlled study of exemestane with or without entinostat in postmenopausal women with locally recurrent or metastatic estrogen receptor-positive breast cancer progressing on treatment with a nonsteroidal aromatase inhibitor. Clin Oncol 2013; 31:2128-35. doi: 10.1200/ JCO.2012.43.7251

32. Lauring J, Park BH, Wolff AC. The phosphoinositide3-kinase-Akt-mTOR pathway as a therapeutic target in breast cancer. NCCN 2013; 11:670-78.

33. Migliaccio I, Di Leo A, Malorni L. Cyclin-dependent kinase 4/6 inhibitors in breast cancer therapy. Curr Opin Oncol 2014; 26:568-75. doi: 10.1097/ CCO.0000000000000129

34. Baselga J, Campone M, Piccart M, et al. Everolimus in postmenopausal hormone-receptor-positive advanced breast cancer.NEJM 2012;366:520-9. doi: 10.1056/ NEJMoa1109653

35. Finn RS, Crown JP, Lang I, et al. The cyclin-dependent kinase $4 / 6$ inhibitor palbociclib in combination with letrozole versus letrozole alone as first-line treatment of oestrogen receptor-positive, HER2-negative, advanced breast cancer (PALOMA-1/TRIO-18): a randomised phase 2 study. The Lancet Oncology 2015; 16:25-35. doi: 10.1016/S1470-2045(14)71159-3

36. Migliaccio I, Malorni L, Hart CD, Guarducci C, Di Leo A. Endocrine therapy considerations in postmenopausal patients with hormone receptor positive, human epidermal growth factor receptor type 2 negative advanced breast cancers. BMC Medicine 2015;13:46. doi: 10.1186/s12916-015-0280-0

37. Treilleux I, Arnedos M, Cropet C, et al. Translational studies within the TAMRAD randomized GINECO trial: evidence for mTORC1 activation marker as a predictive factor for everolimus efficacy in advanced breast cancer. Ann Oncol 2015; 26:120-5. doi: 10.1093/ annonc/mdu497

38. Hortobagyi GNP-GMJ RH, Burris HA, Campone M, Noguchi S, Perez, AT DI, Shtivelband M, Provencher L, Masuda N, Dakhil SR, Anderson I, Chen D, Damask
A, Huang A, McDonald R, Taran T, Sahmoud T, Baselga J. Correlation of molecular alterations with efficacy of everolimus in hormone receptor-positive, HER2-negative advanced breast cancer: Results from BOLERO-2. In: ASCO Annual Meeting. Chicago, IL: J Clin Oncol 31, 2013.

39. Ciruelos Gil EM. Targeting the PI3K/AKT/mTOR pathway in estrogen receptor-positive breast cancer. Cancer Treat Rev 2014; 40:862-71. doi: 10.1016/j. ctrv.2014.03.004.

40. Krop IJS JS, Mayer IA, Dickler M, Ganju V, ForeroTorres A, Melichar B, Morales S, de Boer R, Gendreau S, Derynck M, Lackner M, Spoerke J, Yeh R-F, Levy G, Ng V, O’Brien C, Savage H, Xiao Y, Wilson T, Lee SC, Petrakova K, Vallentin S, Yardley D, Ellis M, Piccart M, Perez EA, Winer E, Schmid P. The FERGI phase II study of the PI3K inhibitor pictilisib (GDC-0941) plus fulvestrant vs fulvestrant plus placebo in patients with $\mathrm{ER}+$, aromatase inhibitor (AI)-resistant advanced or metastatic breast cancer - Part I results. In: San Antonio Breast Cancer Symposium. San Antonio, Texas; 2014.

41. Dhillon S: Palbociclib: first global approval. Drugs 2015; 75:543-51. doi: 10.1007/s40265-015-0379-9.

42. Gelbert LM, Cai S, Lin X, Sanchez-Martinez C, et al. Preclinical characterization of the CDK4/6 inhibitor LY2835219: in-vivo cell cycle-dependent/independent anti-tumor activities alone/in combination with gemcitabine. Invest New Drug 2014; 32:825-37. doi: 10.1007/s10637-014-0120-7.

43. Tate SC, Cai S, Ajamie RT, et al. Semi-mechanistic pharmacokinetic/pharmacodynamic modeling of the antitumor activity of LY2835219, a new cyclindependent kinase 4/6 inhibitor, in mice bearing human tumor xenografts. Clin Cancer Res 2014; 20:3763-74. doi: 10.1158/1078-0432.CCR-13-2846

44. Infante JR SG, Witteveen P, Gerecitano JF, et al. A phase I study of the single-agent CDK4/6 inhibitor LEE011 in pts with advanced solid tumors and lymphomas. In: ASCO Annual Meeting. Chicago, IL. J Clin Oncol 2014. 